\title{
Task-related brain oscillations in normal aging*
}

\author{
Ming-Chung Ho ${ }^{1}$, Chin-Fei Huang ${ }^{2}$, Chia-Yi Chou ${ }^{1,3}$, Yu-Te Lin ${ }^{4}$, Ching-Sen Shih ${ }^{2,4}$, \\ Ming-Ting Wu ${ }^{5,6}$, Chao-Ming Hung ${ }^{2,7}$, Chia-Ju Liu ${ }^{2 \#}$ \\ ${ }^{1}$ Department of Physics, National Kaohsiung Normal University, Kaohsiung, Chinese Taipei \\ ${ }^{2}$ Graduate Institute of Science Education, National Kaohsiung Normal University, Kaohsiung, Chinese Taipei; \\ \#Corresponding Author: chiaju1105@gmail.com \\ ${ }^{3}$ Department of Environmental and Occupational Safety and Hygiene, Graduate Institute of Environmental Management, Tajen \\ University, Pingtung, Chinese Taipei \\ ${ }^{4}$ Section of Neurology, Kaohsiung Veterans General Hospital, Kaohsiung, Chinese Taipei \\ ${ }^{5}$ Faculty of Medicine, National Yang-Ming University, Shipai, Chinese Taipei \\ ${ }^{6}$ Section of Radiology, Kaohsiung Veterans General Hospital, Kaohsiung, Chinese Taipei \\ ${ }^{7}$ Section of General Surgery, E-DA Hospital, Kaohsiung, Chinese Taipei
}

Received 27 July 2012; revised 25 August 2012; accepted 5 September 2012

\begin{abstract}
During the past few years, many researchers have demonstrated the importance of the agerelated changes in spontaneous electroencephalography. However, very little research on of the event-related responses of oscillations connections has been used to examine the changes during normal aging. The aim of the present study was to investigate age-related changes of task-related brain oscillations, which include spectral power and omega-complexity. We hypothesized that the power and omega-complexity of the brain are affected by age-related changes, which could be observed in this study. The samples included young and healthy elderly groups. Compared to young participants, elderly participants were found to have increased power in anterior area and decreased power in posterior area, and have shown a decreased power in the alpha-1 $(7-10 \mathrm{~Hz})$ and alpha-2 $(10-13 \mathrm{~Hz})$ bands and an increased power in the delta (1 - 4 $\mathrm{Hz}$ ) band. Elderly participants were found to have increased omega-complexity in the anterior and posterior brain areas, and have shown an increased omega-complexity in the alpha-2, beta-1 $(13-18 \mathrm{~Hz})$, and beta-2 $(18-30 \mathrm{~Hz})$ bands. The findings in this study suggest that power and omega-complexity changes in task-specific neural activity may potentially be used to assess age-related decline in the brain.
\end{abstract}

Keywords: Normal Aging; Power; Omega-Complexity

\footnotetext{
"The authors have no conflict of interest.
}

\section{INTRODUCTION}

During the past years, numerous studies have demonstrated the importance of the spatial synchronization of the oscillators in spontaneous electroencephalography (EEG) $[1,2]$. A previous study of spontaneous EEG, using spectral power analysis, found the delta, alpha-1, and alpha-2 bands to be higher in young people than in healthy aging people [1]. Another study reported that omega-complexity values were higher in healthy aging people than in young people [3]. However, these studies have mainly depended on spontaneous EEG studies of different parts of the brain while their participants were recorded with eyes closed and eyes opened resting state conditions. The earlier study of spontaneous brain oscillatory activity in a resting state has provided useful information for aging-related research, yet a more comprehensive explanation of real life evocation of brain oscillations is needed in event-related potential (ERPs) studies. One of the main focuses of previous EEG and ERP studies lies on pathological oscillatory communication [4], which showed that greater attention is being paid to evoke oscillatory activity in the brain while performing cognitive tasks for assessing disease-related neurophysiologic change. Hence, this study focuses on task-related brain oscillations to investigate the age-related features in healthy aging people.

Previous studies have also demonstrated that analysis of neuronal oscillations can help in understanding the synchronized activity of neural connectivity [2]. In the last decades, measurement of spontaneous EEG in normal aging has demonstrated that alpha power reduction was significant at each electrode but was most prominent at the parietal site [5]. However, it is not easy to show the complex connections and oscillations of brain signals only by spontaneous EEG analysis. Then, to highlight 
the spatial reorganization of the oscillatory activity in the presence of simple auditory stimulus with respect to the background activity, event-related potentials (ERPs) study of brain connectivity is expected to be useful in understanding the normal aging [6]. For this reason, to evoke task-related oscillations, the auditory oddball paradigm was used. Both spectral power (single electrode) and omega-complexity (local areas) analyses performed with respect to the target stimulus. The auditory oddball task is frequently used in electrophysiological research, which is thought to reflect the "focused attention", "target recognition", "motor response", "working memory", and "decision making" [7] aspects of cognitive performance. ERPs provide a noninvasive electrophysiological measure of brain activity. The findings from past studies have indicated that healthy aging people revealed increased P300 amplitude and decreased P300 latency in responding to the auditory oddball task [8,9]. However, these studies did not attempt to analyze task-related oscillatory activity. Therefore, this study uses task-related spectral power and omega-complexity to explore the changes of frequency bands and oscillatory changes in different brain areas in 15 younger participants and 15 healthy elderly participants when they performed the auditory oddball task. We hypothesize that brain oscillation is affected by age-related changes, which could be reflected in task-related spectral power and omega-complexity. To our knowledge, this study represents the first study of brain oscillation in participants with age-related changes using the analysis of task-related spectral power and omega-complexity.

\section{MATERIALS AND METHODS}

\subsection{Participants}

This study consisted of 30 participants divided into 2 groups: one consisting of 15 young people (mean age $=$ $23.7 \pm 2.8$ years; mean years of education $=16.5 \pm 2.4$; 10 men and 5 women), another of 15 elderly people $(70.1 \pm 7.9$ years; mean MMSE score $29.2 \pm 0.7$; mean years of education $=10.6 \pm 3.5 ; 10$ men and 5 women), with no participants in either group reporting a history of neurologic or psychiatric disease. The protocol of this study was approved by the medical ethics committee of National Kaohsiung Normal University. Written informed consent was obtained from all participants before the experiment. Elderly was evaluated based on the Mini-Mental State Examination [10].

\subsection{Procedure and EEG Recording}

Stimulus presentation was generated by Neuroscan Stim 3.3 Software. Participants were asked to distinguish a target stimulus $(1000 \mathrm{~Hz})$ from a standard stimulus
(2000 Hz), both with sound pressure levels of $85 \mathrm{~dB}$ presented randomly. The probability of each sound category was $80 \%$ for the standard auditory stimulus and $20 \%$ for the target stimulus. Both stimuli were pure tones with $20 \mathrm{~ms}$ duration, including $10 \mathrm{~ms}$ of rise and fall times. The participants were instructed to sit in front of a computer in a soundproof room and listen for sounds emitted by the computer speakers. The participants needed to press a button as soon as they heard a target stimulus, but not a standard stimulus. The participants participated in this experiment for $5 \mathrm{~min}$ and listened to 150 stimuli (30 target tones and 120 standard tones) at 2 $\mathrm{s}$ intervals between each sounding.

The EEG was amplified by the SynAmps/SCAN 4.4 hardware and software (NeuroScan, Inc., Herndon, VA), using the commercial electro-cap (Electro-Cap International, Eaton, $\mathrm{OH}$ ) with electrodes placed at 30 scalp locations (FP1, FP2, F7, F8, F3, F4, FZ, FT7, FC3, FCZ, FC4, FT8, T3, C3, CZ, C4, T4, TP7, CP3, CPZ, CP4, TP8, PZ, P3, P4, T5, T6, O1, OZ, O2) based on the 10 20 system. The reference electrodes were placed in the earlobes. The electrode impedance was maintained below $5 \mathrm{k} \Omega$. The signal was analog filtered and analogto-digital (A/D) converted with a sampling rate of 1000 $\mathrm{Hz}$. Mean reaction time and correct response rate were measured. The signal was filtered (FIR, notch-filtered at $60 \mathrm{~Hz}, 0.1-30 \mathrm{~Hz}, 6 \mathrm{~dB} /$ octave, and zero phase shift) with an epoch length of $200 \mathrm{~ms}$ before and $1800 \mathrm{~ms}$ after stimulus, which included a 200-ms pre-stimulus baseline. Data from single-trial epochs exhibiting excessive movement artifacts $(>90 \mu \mathrm{V})$ were rejected.

\subsection{Data Analysis}

Omega-complexity measure is global method that can analyze signals from all EEG channels simultaneously, while coherence measure is local method that links between pairs of signals [11]. Therefore, omega-complexity measure is applicable to processes that produce EEG signals distributed over the entire scalp. The omegacomplexity quantifies the brain electric field information obtained about the synchronization over the entire scalp [12]. Omega-complexity varies from 1 for a one-dimensional distribution (maximal synchronization) to the number of channels for identically distributed white noise. Thus, greater omega-complexity is indicative of less synchronization or the presence of a poor performance on oddball tasks. In other word, the strength of spatial synchronization decreases with increase of omegacomplexity. Over the past decade of research, this approach has been applied successfully in sleep research [13], sensorimotor control [14], manic-depressive [15], cognitive processes [16], mild Alzheimer's disease [17], epilepsy [18] and age-related features [1], etc. 
Auditory single-sweep potentials were digitally filtered in the 6 frequency bands during target tone processing, and were then analyzed for the anterior, central, and posterior areas. The data were analyzed using EEGLAB version 9.0 [19] under Matlab 7. The statistical data were tested by one-way or two-way analysis of variance (ANOVA) using SPSS 12.0. A p-value of .05 was considered significant.

Frequency analysis was performed in the delta, theta (4 - $7 \mathrm{~Hz}$ ), alpha-1, alpha-2, beta-1, and beta-2 frequency bands (Figure 1). Mean values were calculated in relative spectral power and omega-complexity, and analyses were computed for the 2 groups averaged in the anterior (FP1, FP2, F7, F3, FZ, F4, F8), central (FC3, FCZ, FC4, C3, $\mathrm{CZ}, \mathrm{C} 4, \mathrm{CP} 3, \mathrm{CPZ}, \mathrm{CP} 4)$, and posterior (P3, PZ, P4, T6, $\mathrm{O} 1, \mathrm{OZ}, \mathrm{O} 2)$ regions. There are two main reasons why the data were analyzed from anterior, central and posterior areas in our research. First, prior studies provided clear evidence that the age-related increase in bilateral frontal activity during cognitive tasks is associated with compensatory processes [20-23]. Second, age-related changes in auditory event-related potentials, the N1 and P3 peak amplitude in response to the target stimulus was maximal at the central and parietal regions [24].

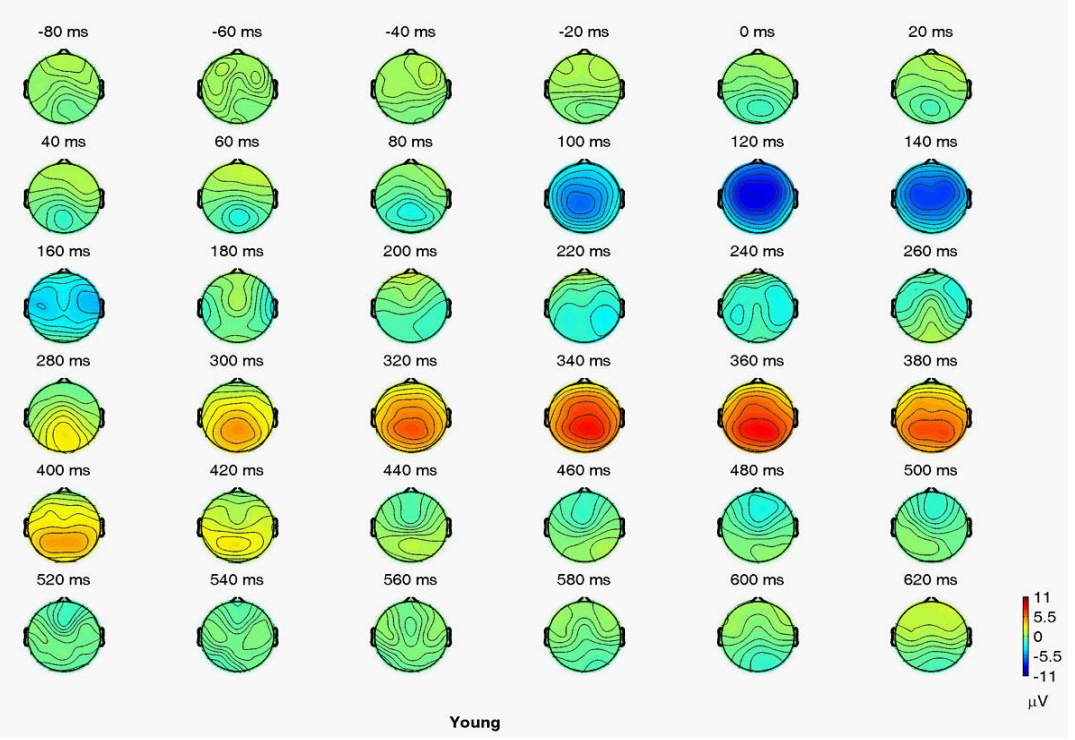

(a)

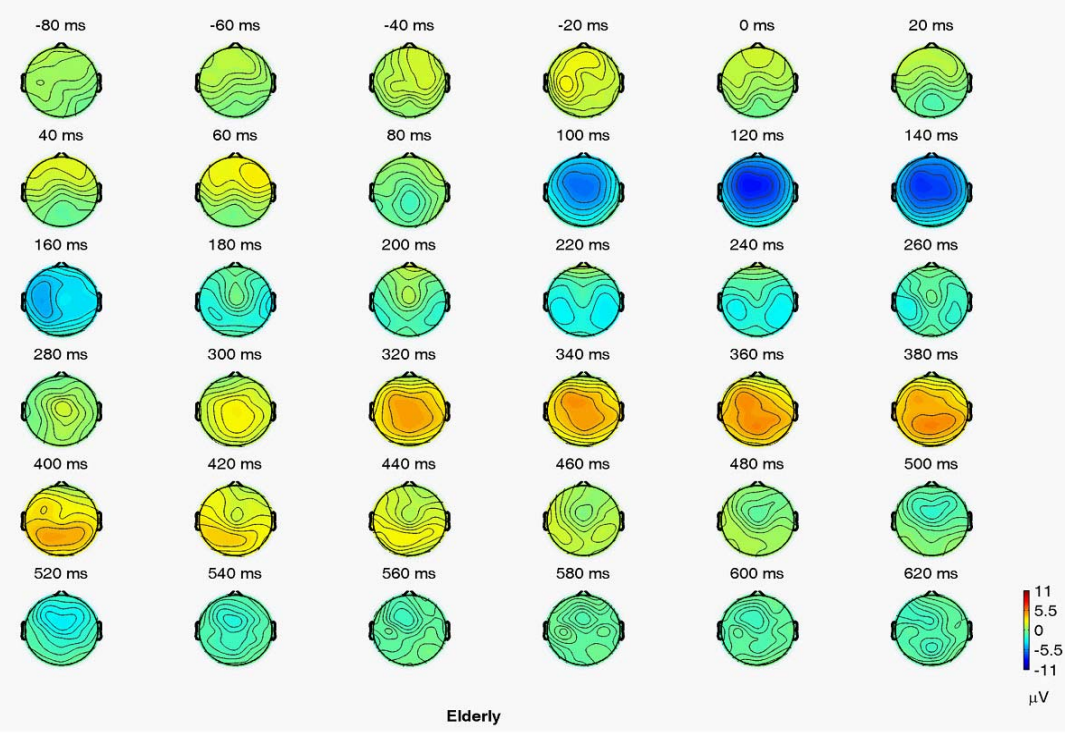

(b)

Figure 1. Grand mean voltage ERP maps for auditory target. (a) Young; (b) Elderly event-related relative spectral power. 


\section{RESULTS}

\section{ERP Results}

The reaction times and response accuracies to target stimuli in an auditory oddball task were $368 \pm 50 \mathrm{~ms}$ and $97.7 \% \pm 6.8 \%$ for the younger group, and $404 \pm 60 \mathrm{~ms}$ and $96.5 \% \pm 5.6 \%$ for the healthy elderly group. As Figure 1 shows, we found the parietal peak amplitudes and peak latency of the young group (P300 amplitude: 10.2 \pm $3.1 \mu \mathrm{V}$; P300 latency: $351 \pm 25 \mathrm{~ms})$ to be higher and shorter than the elderly group (P300 amplitude: $7.2 \pm 2.9$ $\mu \mathrm{V}$; P300 latency: $369 \pm 35 \mathrm{~ms}$ ).

The spectral power values were analyzed to explore the changes of brain oscillation between groups responding to the target stimuli in the various bands. No difference was found between groups in the power [age main effect, $F(1$, $28)=1.32, p=0.251]$ and in the area [area main effect, $\mathrm{F}(1,28)=1.92, \mathrm{p}=0.147]$. Figure 2(a) shows an age group $\times$ area interaction analysis $[\mathrm{F}(2,56)=8.753, \mathrm{p}<$ $0.001]$, which demonstrated that spectral power was significantly higher in the elderly group than in the elderly group in the anterior $(\mathrm{p}=0.012)$ and posterior $(\mathrm{p}=0.001)$ areas. An age group $\times$ frequency interaction analysis $[\mathrm{F}(5$, $140)=4.33, p<0.01]$ in Figure 2(b) demonstrated that spectral power significantly increased in the elderly group than in the young group in the theta $(p=0.008)$, alpha-1 $(p=0.007)$ and alpha-2 $(p=0.004)$ bands.

For omega-complexity analysis, elderly participants demonstrated substantially higher complexity than the young groups [age main effect, $\mathrm{F}(1,28)=18.379$, $\mathrm{p}<$ 0.01]. The omega-complexity was higher in the posterior than in the anterior areas, and in the anterior than in the central area [area main effect, $F(2,28)=73.717, \mathrm{p}<$
0.01]. As Figure 3(a) shows, an age group $\times$ area interaction analysis $[\mathrm{F}(2,56)=2.362, \mathrm{p}=0.095]$ demonstrated that omega-complexity significantly increased in the elderly group than in the young group in the anterior $(p<0.001)$ and posterior $(p<0.001)$ brain areas. The age group $\times$ frequency interaction analysis $[\mathrm{F}(5,140)=$ $1.452, \mathrm{p}=0.204]$ (Figure 3(b) showed that the omegacomplexity value significantly increased in the elderly group than in the young group in the alpha- $2(\mathrm{p}<0.001)$, beta-1 $(\mathrm{p}=0.047)$ and beta- $(\mathrm{p}<0.033)$ frequency bands.

\section{DISCUSSION}

This study found clear differences in power and complexity values in various frequency bands and brain areas between groups through task-related spectral power and omega-complexity. The findings indicating a difference of power in delta, alpha- 1 and alpha- 2 bands, which is in line with previous research $[25,26]$.

Previous study has indicated that aging produces specific changes in the frequency content of the spontaneous EEG. More specifically, an increase in low-frequency power activities has been described [26]. In our study, the healthy elderly group was found by spectral power to have increased power in the delta band compared to young group. The delta response is likely related to signal detection and decision making [7] and the anterior cortical activity is related to target recognition and sustained activity [27]. The results are interpreted that elderly participants may need increased brain activity to respond to target recognition and sustained activity than younger people [6].

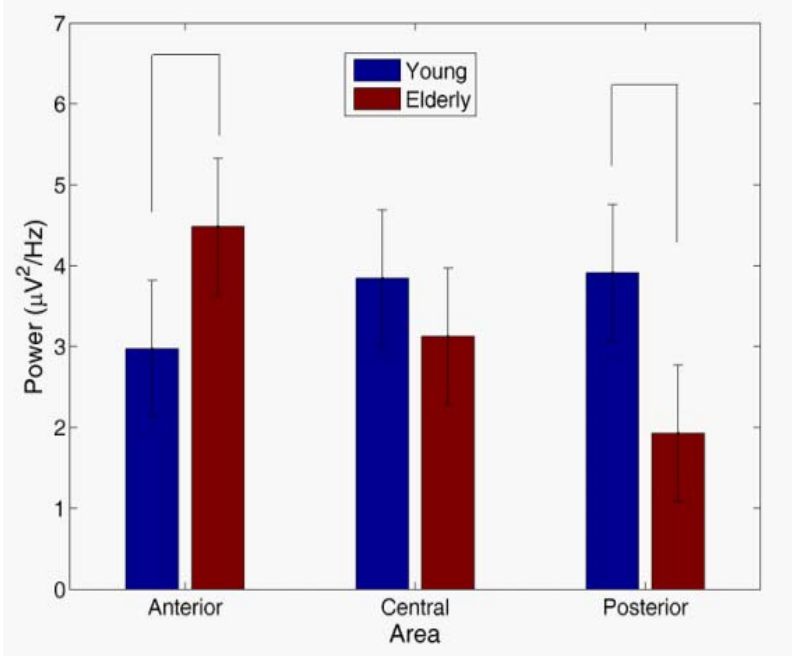

(a)

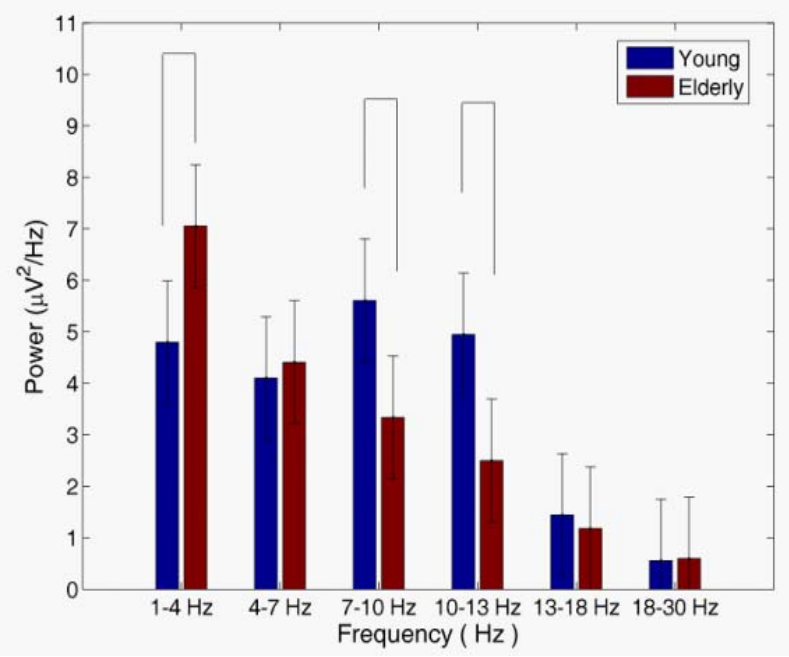

(b)

Figure 2. Age group $\times$ area interaction (a) and age group $\times$ frequency interaction (b) for power. Vertical bars indicate $95 \%$ confidence intervals. Power values in the various frequency bands for the different groups that were significantly different $(p<0.05)$ are connected with horizontal lines at the top of the graph. 


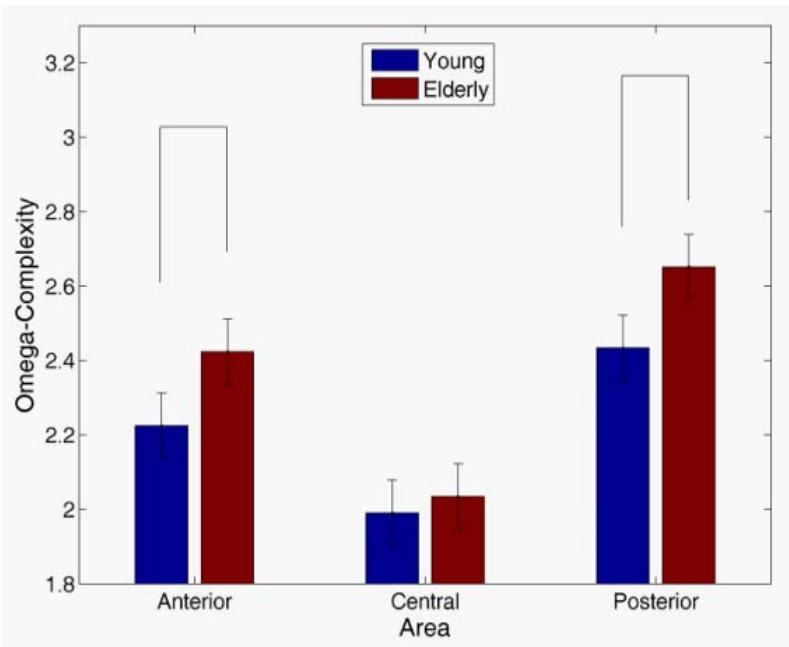

(a)

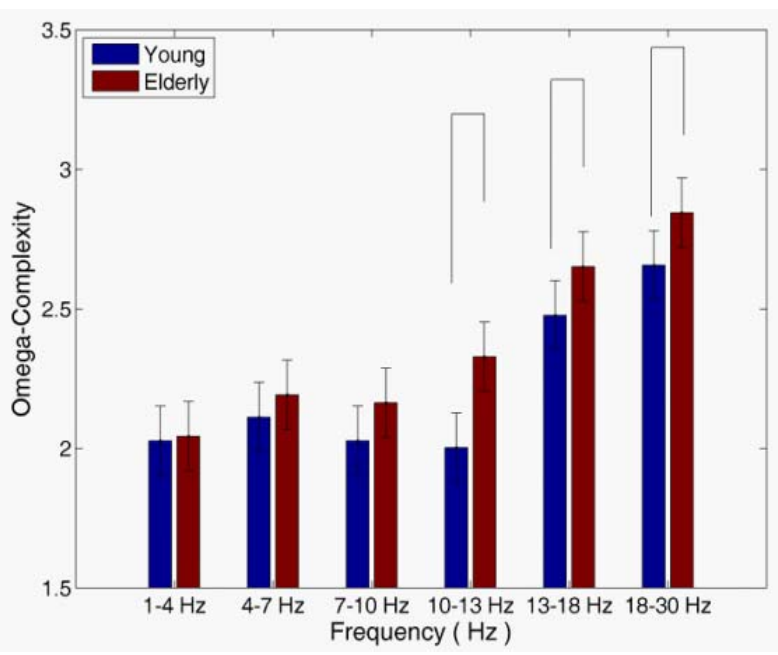

(b)

Figure 3. Age group $\times$ area interaction (a) and age group $\times$ frequency interaction (b) for omega-complexity. Vertical bars indicate $95 \%$ confidence intervals. Omega-complexity values in the various frequency bands for the different groups that were significantly different $(\mathrm{p}<0.05)$ are connected with horizontal lines at the top of the graph.

The previous studies imply that the decrease of EEG alpha power during aging corresponds to power variation in the functional involvement, such as visual performance [28], spatial working memory task [29]. In this study, there was a prominent effect of aging on posterior alpha $(7-13 \mathrm{~Hz})$ oscillations whose power was significantly lower in elderly subjects. The finding is interpreted that elderly participants in aging-related decline may be affected by the slowing information processing over the anterior scalp to respond to target recognition and sustained activity.

In a previous study suggests that mobile phone affect inter-hemispheric synchronization of the EEG alpha rhythms as a function of the physiological aging [30]. Indeed, it is likely that age-related decline in context processing has been associated with decreased synchronization [2]. Compared to young participants, the healthy elderly participants were found by omega-complexity values to have increased values in the anterior and posterior area, and have shown an increased omega-complexity in the alpha-2, beta-1, and beta- 2 bands. Based on the functional connectivity hypothesis [31], the results might reflect that the functional synchronization of young people is more strongly than in healthy elderly people, particularly in the anterior and posterior areas. It is thus not surprising that the synchronous activation induced by perceptual uncertainty and difficulty in decision making, elderly people produce a weakly synchronized frontal alpha oscillation [32].

This study was subject to a limitation. The analysis did not include older participants who displayed poor performance. Such a design allowed us to investigate agerelated changes in cognitive activity to differentiate be- tween good and poor memory task performers. This limitation affects the generalizability of our findings.

\section{CONCLUSION}

This study has clearly demonstrated that task-related spectral power and omega-complexity were affected by aging. Although this study used relatively simple tasks, differences in the auditory task were detected reliably between the age-related groups. Further study using a larger sample size and a greater variety of sampling is needed to investigate the changes of task-related oscillations in the future.

\section{ACKNOWLEDGEMENTS}

The authors would like to thank the National Science Council, Chinese Taipei, for financial support for this research under contract No. NSC 99-2112-M-017-001-MY3 and No. NSC 99-2511-S-017-004MY3. We would also like to thank the personnel of the Neurology section of Kaohsiung Veterans' General Hospital for their advice on this work.

\section{REFERENCES}

[1] Gaál, Z.A., Boha, R., Stam, C.J. and Molnár, M. (2010) Age-dependent features of EEG-reactivity-Spectral, complexity, and network characteristics. Neuroscience Letters, 479, 79-84. doi:10.1016/i.neulet.2010.05.037

[2] Stam, C.J., Breakspear, M., van Walsum, A.-M.V.C. and van Dijk, B.W. (2003) Nonlinear synchronization in EEG and whole-head MEG recordings of healthy subjects. Human Brain Mapping, 19, 63-78. doi:10.1002/hbm.10106

[3] Czigler, I., Cox, T.J., Gyimesi, K. and Horváth, J. (2007 
Event-related potential study to aversive auditory stimuli. Neuroscience Letters, 420, 251-256. doi:10.1016/i.neulet.2007.05.007

[4] Basar, E. and Guntekin, B. (2008) A review of brain oscillations in cognitive disorders and the role of neurotransmitters. Brain Research, 1235, 172-193. doi:10.1016/j.brainres.2008.06.103

[5] Yordanova, J.Y., Kolev, V.N. and Basar, E. (1998) EEG theta and frontal alpha oscillations during auditory processing change with aging. Electroencephalography and Clinical Neurophysiology, 108, 497-505. doi:10.1016/S0168-5597(98)00028-8

[6] Ho, M.C., Chou, C.Y., Huang, C.F., Lin, Y.T., Shih, C.S., Han, S.Y. and Liu, C.J. (2012) Age-related changes of task-specific brain activity in normal aging. Neuroscience Letters, 507, 78-83. doi:10.1016/j.neulet.2011.11.057

[7] Başar-Eroglu, C., Başar, E., Demiralp, T. and Schurmann, M. (1992) P300-response: Possible psychophysiological correlates in delta and theta frequency channels. A review. International Journal of Psychophysiology, 13, 161-179. doi:10.1016/0167-8760(92)90055-G

[8] Polich, J. (1996) Meta analysis of P300 normative aging studies. Psychophysiology, 33, 334-353. doi:10.1111/j.1469-8986.1996.tb01058.x

[9] Polich, J. (1997) On the relationship between EEG and P300: Individual differences, aging, and ultradian rhythms. International Journal of Psychophysiology, 26, 299-317. doi:10.1016/S0167-8760(97)00772-1

[10] Folstein, M., Folstein, S. and McHugh, P. (1975) Minimental state. A practical method for grading the cognitive state of patients for the clinician. Journal of Psychiatric Research, 12, 189. doi:10.1016/0022-3956(75)90026-6

[11] Dauwels, J., Vialatte, F., Musha, T. and Cichocki, A. (2010) A comparative study of synchrony measures for the early diagnosis of Alzheimer's disease based on EEG. Neuroimage, 49, 668-693. doi:10.1016/j.neuroimage.2009.06.056

[12] Wackermann, J. (1996) Beyond mapping: Estimating complexity of multichannel EEG recordings. Acta Neurobiologiae Experimentalis, 56, 197.

[13] Szelenberger, W., Wackermann, J., Skalski, M., Niemcewicz, S. and Drojewski, J. (1996) Analysis of complexity of EEG during sleep. Acta Neurobiologiae Experimentalis, 56, 165.

[14] Stancak, A. and Wackermann, J. (1998) Spatial EEG synchronisation over sensorimotor hand areas in brisk and slow self-paced index finger movements. Brain Topography, 11, 23-31. doi:10.1023/A:1022214402649

[15] Bhattacharya, J. (2000) Complexity analysis of spontaneous EEG. Acta Neurobiologiae Experimentalis, 60, 495-502.

[16] Bhattacharya, J. and Petsche, H. (2001) Musicians and the gamma band: A secret affair? NeuroReport, 12, 371 . doi:10.1097/00001756-200102120-00037

[17] Yoshimura, M., Isotani, T., Yagyu, T., Irisawa, S., Yoshida, T., Sugiyama, M. and Kinoshita, T. (2004) Global approach to multichannel electroencephalogram analysis for diagnosis and clinical evaluation in mild Alzheimer's disease. Neuropsychobiology, 49, 163-166. doi:10.1159/000076724

[18] Kondakor, I., Toth, M., Wackermann, J., Gyimesi, C., Czopf, J. and Clemens, B. (2005) Distribution of spatial complexity of EEG in idiopathic generalized epilepsy and its change after chronic valproate therapy. Brain Topography, 18, 115-123. doi:10.1007/s10548-005-0280-z

[19] Delorme, A. and Makeig, S. (2004) EEGLAB: An open source toolbox for analysis of single-trial EEG dynamics including independent component analysis. Journal of Neuroscience Methods, 134, 9-21. doi:10.1016/j.jneumeth.2003.10.009

[20] Cabeza, R. (2002) Hemispheric asymmetry reduction in older adults: The HAROLD model. Psychology and Aging, 17, 85-100. doi:10.1037//0882-7974.17.1.85

[21] Cabeza, R., Anderson, N.D., Locantore, J.K. and McIntosh, A.R. (2002) Aging gracefully: Compensatory brain activity in high-performing older adults. Neuroimage, 17, 1394-1402. doi:10.1006/nimg.2002.1280

[22] Grady, C.L. (1998) Brain imaging and age-related changes in cognition. Experimental Gerontology, 33, 661-673. doi:10.1016/S0531-5565(98)00022-9

[23] Phillips, L.H. and Andrés, P. (2010) The cognitive neuroscience of aging: New findings on compensation and connectivity. Cortex, 46, 421-424. doi:10.1016/j.cortex.2010.01.005

[24] Pfefferbaum, A., Wenegrat, B.G., Ford, J.M., Roth, W.T. and Kopell, B.S. (1984) Clinical application of the P3 component of event-related potentials. II. Dementia, depression and schizophrenia. Electroencephalography and Clinical Neurophysiology/Evoked Potentials Section, 59, 104-124

[25] Klimesch, W. (1999) EEG alpha and theta oscillations reflect cognitive and memory performance: A review and analysis. Brain Research Reviews, 29, 169-195. doi:10.1016/S0165-0173(98)00056-3

[26] Polich, J. (1997) EEG and ERP assessment of normal aging. Electroencephalography and Clinical Neurophysiology/Evoked Potentials Section, 104, 244-256.

[27] Miller, E.K., and Cohen, J.D. (2001) An integrative theory of prefrontal cortex function. Annual Review of Neuroscience, 24, 167-202. doi:10.1146/annurev.neuro.24.1.167

[28] Kononen, M. and Partanen, J. (1993) Blocking of EEG alpha activity during visual performance in healthy adults. A quantitative study. Electroencephalography and Clinical Neurophysiology, 87, 164-166. doi:10.1016/0013-4694(93)90122-C

[29] McEvoy, L.K., Pellouchoud, E., Smith, M.E. and Gevins, A. (2001) Neurophysiological signals of working memory in normal aging. Cognitive Brain Research, 11, 363376. doi:10.1016/S0926-6410(01)00009-X

[30] Vecchio, F., Babiloni, C., Ferreri, F., Buffo, P., Cibelli, G., Curcio, G. and Rossini, P.M. (2010) Mobile phone emission modulates inter-hemispheric functional coupling of EEG alpha rhythms in elderly compared to young subjects. Clinical Neurophysiology, 121, 163-171. doi:10.1016/j.clinph.2009.11.002 
[31] Breznitz, Z. (2003) Speed of phonological and orthographic processing as factors in dyslexia: Electrophysiological evidence. Genetic, Social, and General Psychology Monographs, 129, 183-206.
[32] Kolev, V., Yordanova, J., Schürmann, M. and Baţar, E. (1999) Event-related alpha oscillations in task processing. Clinical Neurophysiology, 110, 1784-1792. doi:10.1016/S1388-2457(99)00105-4 